\title{
Research on Evaluation Method of Video Stabilization
}

\author{
Bin Chen, Jianjun Zhao and Yi Wang \\ Weapon Science and Technology Dept, NAAU, Yantai, China
}

\begin{abstract}
In order to accomplish the task of evaluating video stabilization, we divided videos into four classes according to the state of the cameras and moving targets in the monitoring areas. It also analyzed the difficulties of evaluating video stabilization in each condition. As PSNR(peak signal to noise ratio) becomes disabled with moving cameras, we proposed a novel evaluation method based on the VIA(variation of intersection angles) between global motion vectors. In order to decrease the influences to PSNR caused by moving objects in FOV(field of view), we computed PSNR of the overlapping background instead of the whole image achieved by fixed camera. As moving objects also affect VIA, we deleted the motion vectors on them and removed the related interference. As the experiments show, the evaluating architecture proposed in this paper can remove the disturbance caused by moving objects in every class of video and evaluate video stabilization correctly.
\end{abstract}

Keywords-video stabilization; evaluation method; global motion vector; VIA

\section{INTRODUCTION}

Video stabilization technology have a wide range of use in surveying, photography and military fields ${ }^{[1,2]}$. Particularly in the military field, the carriers on which the image sensors mounted are mostly mobile. The vibration and jitter of the carriers will lead to instability of the captured video. As the unstable video have a greater negative effect on the subsequent stages like image processing, video stabilization technology has become a hot field of image processing.

The current evaluating methods of video stabilization are mainly based on the PSNR (peek signal-to-noise ratio). PSNR can only get better results in videos captured by fixed cameras without moving objects. In other words, when moving targets appear in the FOV(field of view) or the camera is mobile, the effect of PSNR would have deviations. In order to solve these problems above, we have accomplished the follow works. Firstly, in the condition with camera fixed, we used the overlapping area of the images instead of entire one to calculate PSNR, thereby removing the effects of the moving targets in the FOV. Secondly, we proposed VIA (variation of intersection angles) method to measure the stability of the video. By selecting the motion vectors in the FOV, the interference caused by moving objects was removed.

\section{VIDEO STABILIZATION TECHNOLOGY}

Video stabilization system can be classified into three categories according to the mechanisms.

- Mechanical stabilization

- Optical stabilization

- Electronic stabilization
The first two kinds are relates to the physical equipment, mainly used by professional photographers and camera manufacturers ${ }^{[3]}$. With the rapid development of digital image processing, stabilize the videos by digital image processing method has become a popular trend. Electronic (digital) stabilization is based on the estimation of motion vectors between successive video frames. Stabilized videos are obtained by doing motion filtering and compensating to each frame in the video.

\section{A. Electronic Stabilization Algorithm}

Electronic stabilization algorithm adopts image processing method to stabilize the video. The algorithm calculates the global motion vector between two continuous frames, filters out the jitter with high frequency, compensates motions to the second frame and finally completes the video stabilization. In summary, the process of video stabilization can be divided into three stages:

- Global motion estimation

- Motion compensation

- Image generation

\section{B. Classification of Video}

The unstable videos can be divided into four categories depending on whether the camera is fixed and whether there are moving objects in the scene or not.

a) Camera fixed without moving objects. In this case, the primary mission is to remove the jitter caused by external factor. PSNR can achieve a good effect.

b) Camera fixed with moving objects. In this case, PSNR will lead to a certain deviation from the actual situation. The deviation is related to the state of the moving objects such as the proportion they occupied in the image and their velocity.

c) Mobile camera without moving object. When the camera is mounted on a mobile carrier, the background of the images in the obtained video will have various changes. Using PSNR will mostly get the wrong evaluating results. The frames which have no relative motion are easier to stabilize.

d) Mobile camera with moving objects. In this case, the frames in obtained video are more complex. We need to consider the movements of the foreground as well as the background, focusing on how to remove the passive effect caused by the moving objects in the foreground.

\section{EVALUATING METHODS OF VIDEO STABILIZATION}

As videos captured in four conditions above have different characteristics, therefore different approaches are needed to do evaluations. If the camera is still, PSNR measurement has a good effect. When there are moving objects in the scene, 
removing the region with them and calculating PSNR in overlapping area can effectively remove the interference caused by moving targets. The shake of the carrier will transmit to the video captured by the camera mounted on. Therefore, VIA between continuous frames is introduced to evaluating the stabilization. However, when moving objects appear in the FOV, the difficulty of obtaining the global motion vector accurately increases dramatically.

\section{A. PSNR Evaluating Algorithm}

After compensating all motions, the best result is that the value of each pixel in subtraction image equals to zero. But the errors, miscalculations and distortion caused by the models may lead to nonzero value. To measure the effect of stabilization, peak signal to noise ratio (PSNR) ${ }^{[4-7]}$ which calculates the degree of deviation from the optimum results is used. The higher the PSNR, the higher accuracy of the system ${ }^{[8]}$. In other words, the PSNR between two same images will be taken to the maximum.

PSNR between two images is defined as Eq. 1.

$$
\operatorname{PSNR}\left(E_{1}, E_{0}\right)=10 \log \frac{2 \mathrm{gs}}{\operatorname{MSE}\left(I_{1}, L_{0}\right)}
$$

The gray level of the image equals to $255, M S E\left(E_{1}, E_{0}\right)$ is the mean square error between two images as Eq. 2 shows.

$$
\operatorname{MSE}\left(E_{1}, E_{0}\right)=\frac{1}{N M} \sum_{n=1}^{N} \sum_{m=1}^{U}\left[I_{1}\left(x_{n}, y_{m}\right)-I_{0}\left(x_{n}^{n}, y_{m}\right)\right]^{2} .
$$

\section{B. Global Motion Vector}

During estimating the global motion vectors, the motion model of the camera is an important issue. All pixels in an image obey a same law if the motion model is determined.

We calculate the global motion vector based on SIFT feature points. Because they are based on several local points of interest and have nothing to do with the size and rotation of the image. They perform very well when noise exists in the image, or view-angle and illumination changes.

SIFT algorithm ${ }^{[9,10]}$ mainly consists of four stages.

a) Extrema detection in scale-space.

b) Key points positioning

c) Direction determining

d) Description of key points

\section{Variation of Intersection Angles(VIA)}

In dynamic background, PSNR cannot correctly measure the stability of video. So we propose a new method called VIA to measure the stability of the video in such conditions.

Suppose there are $n$ frames in a video defined as $f_{1} f_{2}, \cdots, f_{\mathrm{m}}$. The obtained global motion vectors are $\overrightarrow{r_{1}}, \overrightarrow{r_{2}}, \cdots, \overrightarrow{r_{n-1}}$. Taking $\overrightarrow{r_{1}}$ and $\overrightarrow{r_{n-1}}$ into account, we defined an initial vector $\overrightarrow{r_{0}}$ and a terminal vector $\overrightarrow{r_{n}}$, which are unit vectors along the positive $\mathrm{x}$-axis, namely:

$$
\overrightarrow{r_{0}}=\overrightarrow{r_{1}}=\overrightarrow{(0,1)}
$$

$\Theta=\left\{\theta_{1}, \theta_{2}, \cdots, \theta_{m}\right\}$ is the set of angles between the adjacent motion vectors.

When the trajectory of global motion vectors has a greater change, the sum of angles $s$ is able to characterize the fluctuation. A larger $s$ indicates a video which is more unstable.

Suppose $s_{k}$ is the VIA set of first $k$ frames, such as the Eq. 4 shows.

$$
s_{k}=\left\{\left\|\theta_{1}\right\|,\left\|\theta_{2}\right\|, \cdots,\left\|\theta_{k}\right\|\right\}
$$

The mean and variance of $s_{k}$ are computed to characterize the stability of first $k$ frames, such as the Eq. 5 and Eq. 6 show.

$$
\begin{gathered}
M s_{k}=\frac{1}{k} \sum_{i=1}^{k}\left\|\theta_{i}\right\| . \\
V s_{k}=\sum_{i=1}^{k}\left(\left\|\theta_{i}\right\|-M s_{k}\right)^{2} .
\end{gathered}
$$

\section{EXPERIMENTS AND RESULTS}

\section{A. Experiment 1}

In the fisrt experiment, the camera mounted at a fixed position. At the same time, there is no moving object in the FOV. We selected 4 consecutive frames in the video(frame 101 to 104). We stabilize the video by a method based on SIFT features. Differential images before and after stabilization are shown in Fig. 1 and Fig. 2.

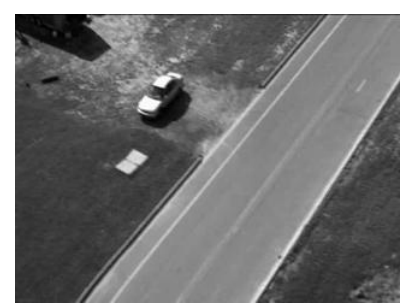

(a) frame 101

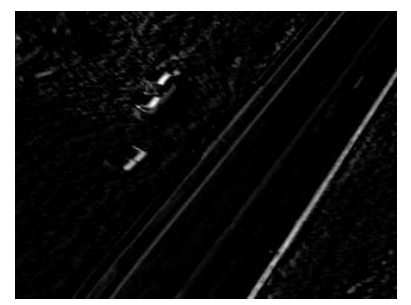

(c) Difference of frame 101 and 103

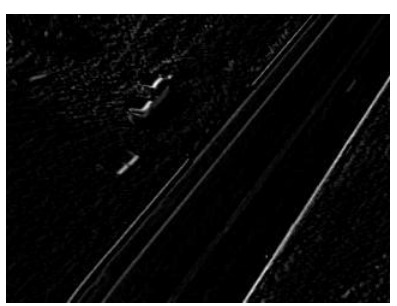

(b) Difference of

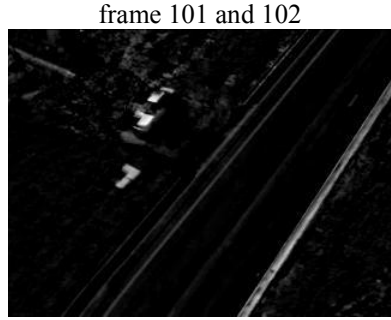

(d) Difference of frame 101 and 104
FIGURE I. TESTING IMAGES OF EXPERIMENT 1 


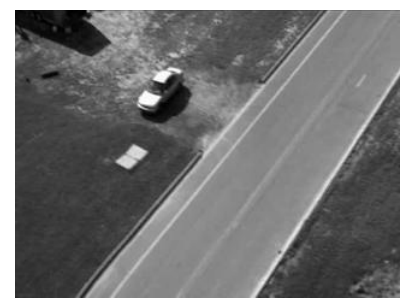

(a) frame 101

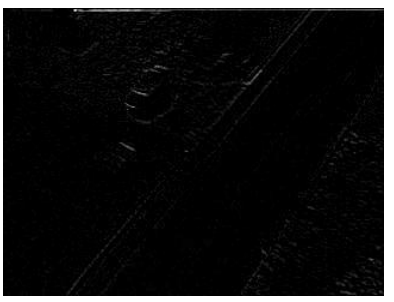

(c) Difference of frame 101 and 103

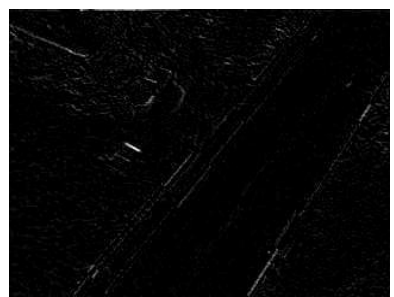

(b) Difference of

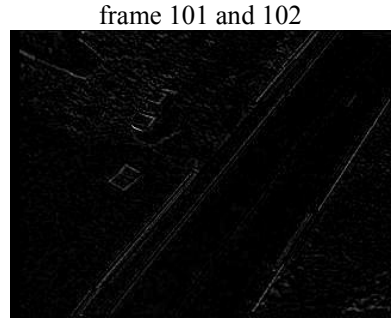

(d) Difference of frame 101 and 104
FIGURE II. DIFFERENCE OF IMAGES AFTER STABILIZATION

We computed the PSNR of two groups of frames, which are given in Table 1 and Table 2.

TABLE I. PSNR OF TESTING IMAGES IN EXPERIMENT 1

\begin{tabular}{|c|c|c|c|c|}
\hline frame & 101 & 102 & 103 & 104 \\
\hline 101 & $\infty$ & 32.85 & 32.01 & 31.29 \\
\hline 102 & 32.85 & $\infty$ & 32.62 & 31.63 \\
\hline 103 & 32.01 & 32.62 & $\infty$ & 32.19 \\
\hline
\end{tabular}

TABLE II. PSNR OF STABILIZED IMAGES

\begin{tabular}{|c|c|c|c|c|}
\hline frame & 101 & 102 & 103 & 104 \\
\hline 101 & $\infty$ & 37.89 & 38.01 & 35.69 \\
\hline 102 & 37.89 & $\infty$ & 35.23 & 34.36 \\
\hline 103 & 38.01 & 35.23 & $\infty$ & 34.2393 \\
\hline 104 & 35.69 & 34.36 & 34.2393 & $\infty$ \\
\hline
\end{tabular}

As Fig. 2 and Fig. 3 shows, the difference between adjacent frames has significantly reduced after stabilization. As shown in Table 1 and Table 3, PSNR between any two frames have been improved, which is consistent with the actual situation.

\section{B. Experiment 2}

When the camera is fixed and moving objects appear in the FOV, the moving targets will result in a reduction of PSNR values. For a fixed camera, the backgrounds of the images are more appropriate to characterize the stability of video, because moving targets brought interference to the PSNR.

The test set of experiment 2 as shown in Fig. 4, which are six frames cut out from a traffic surveillance video. As shown, there are several vehicles moving in the FOV. The PSNR calculated are shown in Table 3.
TABLE III. PSNR OF TESTING IMAGES IN EXPERIMENT 2

\begin{tabular}{|c|c|c|c|c|c|c|}
\hline frame & 1 & 2 & 3 & 4 & 5 & 6 \\
\hline 1 & $\infty$ & 40.26 & 38.79 & 37.98 & 37.36 & 35.44 \\
\hline 2 & 40.26 & $\infty$ & 40.49 & 38.73 & 38.19 & 35.6 \\
\hline 3 & 38.79 & 40.49 & $\infty$ & 40.21 & 36.98 & 35.03 \\
\hline 4 & 37.98 & 38.73 & 40.21 & $\infty$ & 36.75 & 34.82 \\
\hline 5 & 37.36 & 38.19 & 36.98 & 36.75 & $\infty$ & 37.79 \\
\hline 6 & 35.44 & 35.6 & 35.03 & 34.82 & 37.79 & $\infty$ \\
\hline
\end{tabular}

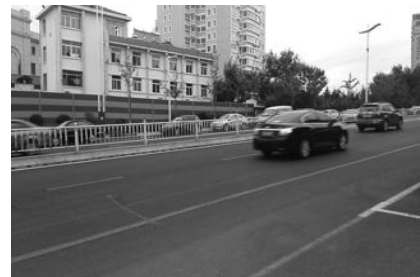

(a) frame 1

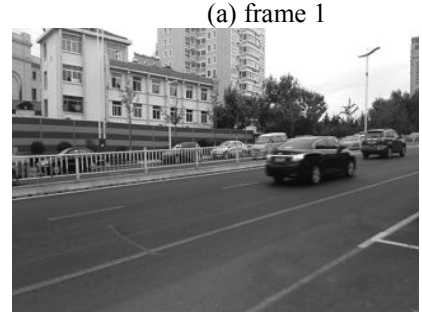

(c) frame 3

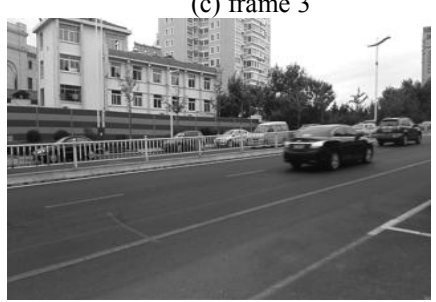

(e) frame 5

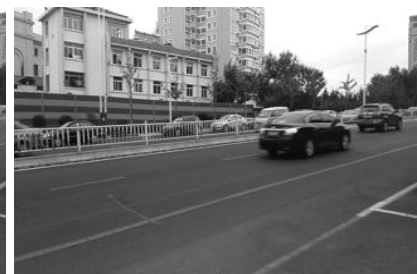

(b) frame 2

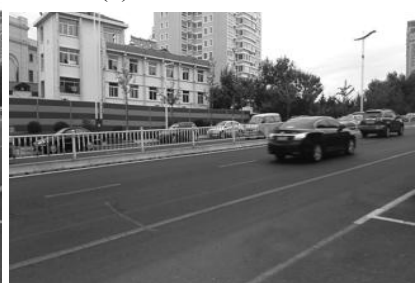

(d) frame 4

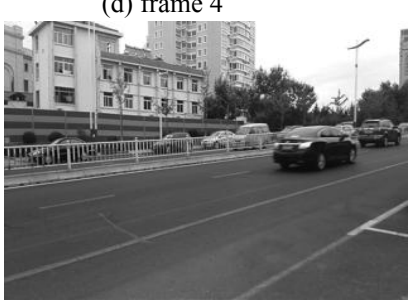

(f) frame 6
FIGURE III. TESTING IMAGES OF EXPERIMENT 2

To rule out the influence of moving targets, we firstly apply detecting algorithm to acquire the area contains the moving objects. The overlapping background can be acquired by taking the area with moving targets out. Then in order to improve the accuracy, the overlapping backgrounds are chose to compute the PSNR. Fig. 4 shows the overlapping backgrounds. PSNR of the overlapping backgrounds are shown in table 4. Obviously, foreground moving targets has some interference to the evaluation of stabilization, especially in the case that the objects occupy a large proportion of the image.

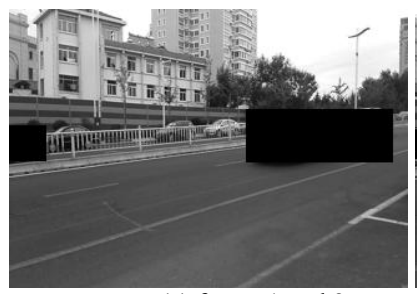

(a) frame 1 and 2

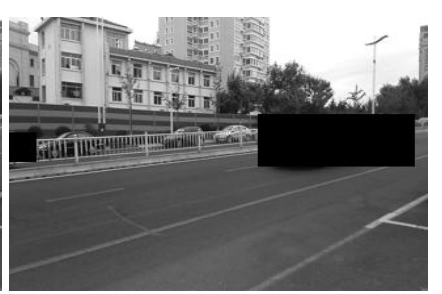

(b) frame 2 and 3 


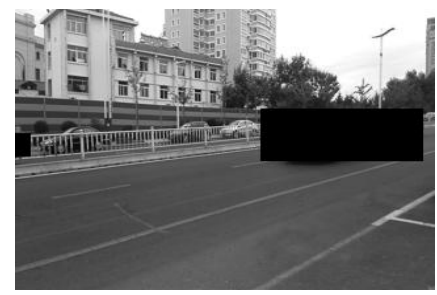

(c) frame 3 and 4

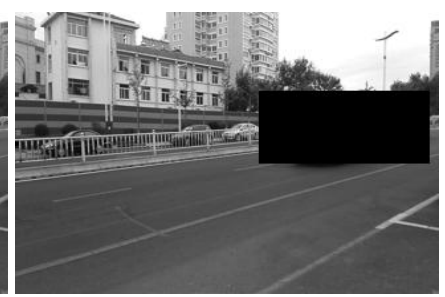

(d) frame 4 and 5

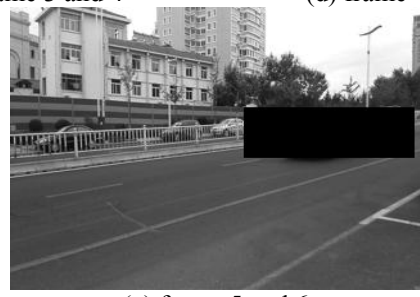

(e) frame 5 and 6

FIGURE IV. OVERLAPPING AREA OF BACKGROUND

TABLE IV. PSNR OF OVERLAPPING AREA OF BACKGROUNDS

\begin{tabular}{|c|c|c|c|c|c|c|}
\hline frame & 1 & 2 & 3 & 4 & 5 & 6 \\
\hline 1 & $\infty$ & 47.01 & 41.91 & 40.05 & 40.53 & 37.88 \\
\hline 2 & 47.01 & $\infty$ & 43.12 & 39.64 & 40.57 & 37.34 \\
\hline 3 & 41.91 & 43.12 & $\infty$ & 42.18 & 39.03 & 36.51 \\
\hline 4 & 40.05 & 39.64 & 42.18 & $\infty$ & 38.97 & 36.41 \\
\hline 5 & 40.53 & 40.57 & 39.03 & 38.97 & $\infty$ & 40.02 \\
\hline 6 & 37.88 & 37.34 & 36.51 & 36.41 & 40.02 & $\infty$ \\
\hline
\end{tabular}

\section{Experiment 3}

If the camera is mounted on a moving carrier, the backgrounds of the video will begin to change variously. At the same time, the accuracy of the PSNR decreased significantly. Using PSNR to evaluate the stability of video may lead to serious error. However, global motion vectors are feasible to measure the stability of video instead of PSNR.

The test set of experiment 3 are shot at a crossing artificially. In order to highlight the effect of the experiment, one video is shot smoothly and the other one have regular swing in the vertical direction. The obtained trajectories of global motion vectors are shown in Fig. 5. Obviously, the trajectory of the stable video is more stable and smooth than the unstable one.

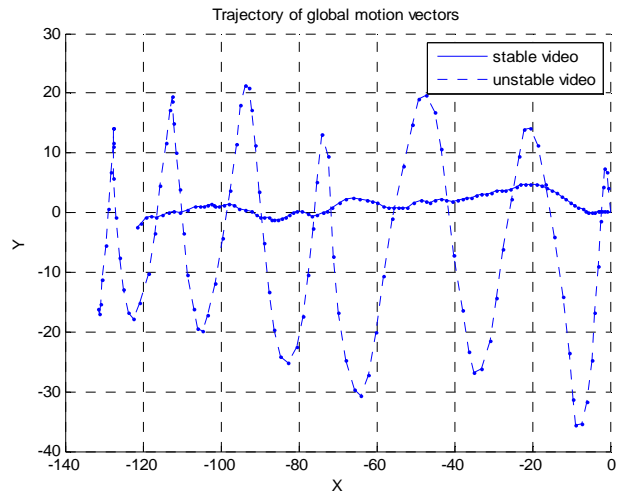

FIGURE V. TRAJECTORIES OF GLOBAL MOTION VECTORS
The curve of mean value of VIA are shown in Fig. 6, showing that the mean of VIA in the stable video varies little along with the increase of frames, and becomes to a convergence. Relatively, the mean of VIA shakes when the unstable video shakes. At the same time, the variation of variances is similar as the situation of mean shown in Fig. 7.

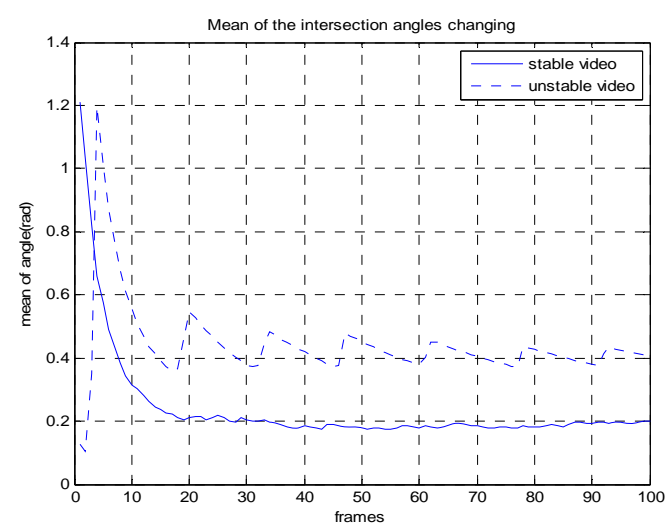

FIGURE VI. MEAN OF THE VIA BETWEEN GLOBAL MOTION VECTORS

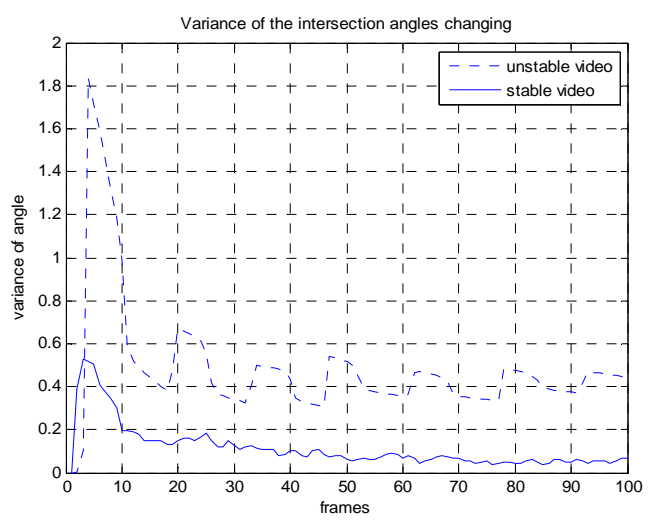

FIGURE VII. VARIANCE OF THE VIA BETWEEN GLOBAL MOTION VECTORS

\section{Experiment 4}

The accuracy of global motion vector is the most important to ensure the effect of the proposed method based on VIA. The feature points locating on the moving objects in the FOV may cause the global motion vector away from the reality. So removing the feature vectors on moving objects can acquire global motion vector more accurate.

Experiment 4 uses 10 successive frames in a traffic surveillance video, which contains several moving cars in the FOV with camera not fixed. In this paper, SIFT feature points are adopted. The first two images of the test set are shown in Fig. 8. After converting the image to gray-scale, extracting the features and matching the feature points, feature vectors without selection are acquired, which are shown in Fig. 9. 


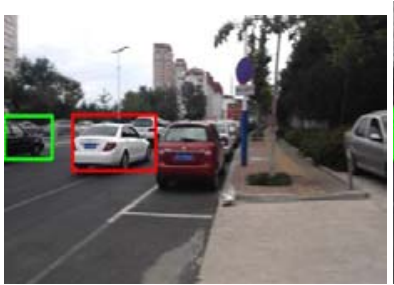

(a) frame 1

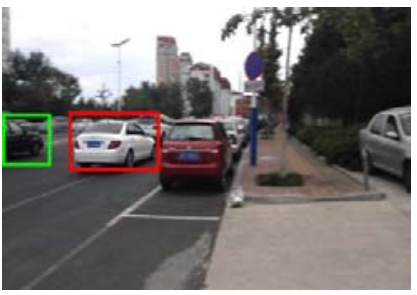

(b) frame 2
FIGURE VIII. 1ST AND 2ND TESTING IMAGES OF EXPERIMENT 4

The motion vectors on moving objects have a greater difference from the one on background. After selection, the motion vectors on the background are acquired finally as shown in Fig. 10.

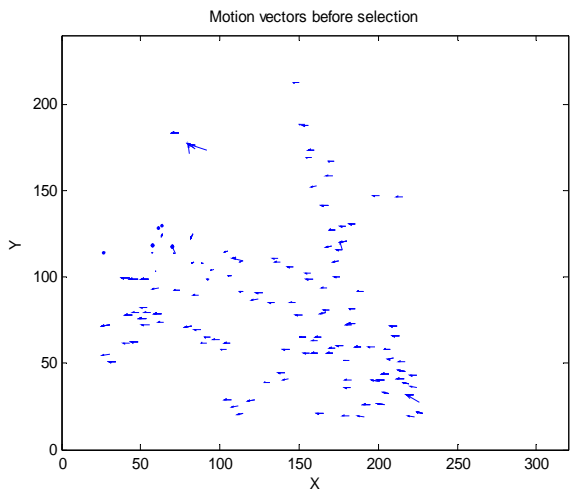

FIGURE IX. MOTION VECTORS OF FEATURE POINTS BEFORE SELECTING

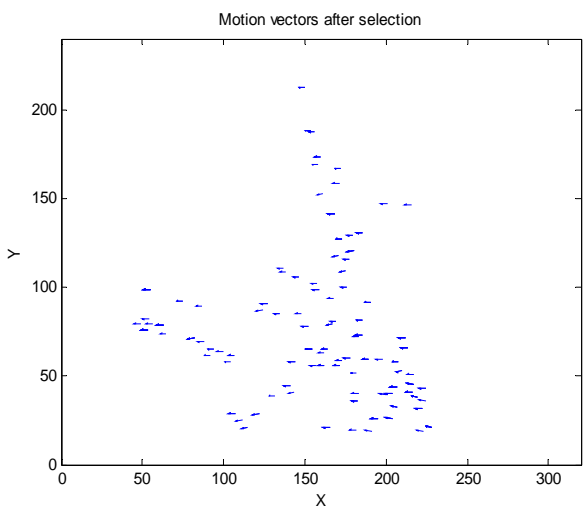

FIGURE X. MOTION VECTORS OF SELECTED FEATURE POINTS

The global motion vectors of the test set obtained are shown in Fig. 11. The vector trajectory before selection is expressed with imaginary line. The full line is the trajectory after selection. Obviously, the vector on the moving objects lead to a trajectory away from the reality. So, removing them can increase the accuracy of the evaluation.

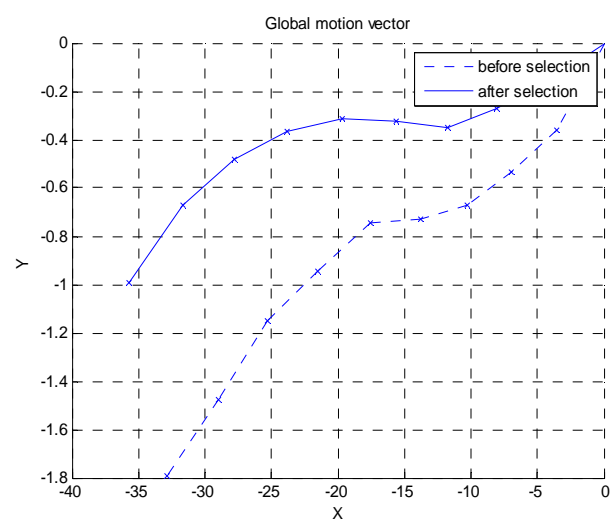

FIGURE XI. COMPARISON OF GLOBAL MOTION VECTORS

\section{CONCLUSION}

In this paper, taking the states of camera and the content of video into account, we divided the acquired videos into four categories. When the camera fixed and there is no moving object in the FOV, PSNR is a good method to evaluate the stability of the video. When moving targets appear, the overlapping background area is used instead of the entire image to calculate PSNR. When the camera are mounted on moving carriers, PSNR is disabled. We proposed a new method called VIA to evaluate the stability of video. As the foreground objects affects accuracy of the global motion vectors, we remove them when moving objects appears.

As experimental results show, PSNR has a good performance in the case that the background is fixed. Moving objects in the scene has some negative influences to PSNR. Choosing the overlapping background as the input of PSNR can effectively remove the interference. When camera is mounted on a moving carrier, PSNR is disabled. The proposed method based on VIA works well in measuring of the stability of videos. Foreground moving objects have interference to the global motion vector, which can be effectively removed by selecting the feature vectors.

In summary, this paper using different methods to evaluate the stability of video according to the type of the video. The evaluation mission can be accomplished in all circumstances.

\section{REFERENCES}

[1] SACHS D, NASIRI S, GOEHL D. Image Stabilization Technology Overview[M]. Santa Clara, CA: InvenSense Inc., 2007: 18.

[2] CHANG H C, LAI S H, LU K R. A Robust Real-time Video Stabilization Algorithm[J]. Journal of Visual Communication and Image Representation, 2006, 17(3): 659-673.

[3] CHEN Qi-li, SONG Li, YU Song-yu. A Overview of Video Stabilization[J]. Video Engineering, 2011,35(7): 15-17.

[4] C. Morimoto and R. Chellappa. Evaluation of image stabilization algorithms[C]. IEEE Int'l Conf. Acoustics, Speech and Signal Processing, 1998, pp. 2789-2792.

[5] FARId H, WOODWARD J B. Video Stabilization and Enhancement[R]. Technical Report TR2007-605, [S.1.] Department of Computer Science, Dartmouth College, USA, 2007:1-8.

[6] ZHUO Lei. Study of Electronic Stabilization Techniques for Video Sequences[D]. Tianjin: School of Electronic Information Engineering, Tianjin University, 2010. 
[7] YANG Jin-bao, LIU Chang-chun, HU Shun-bo. Arithmetic Harmonic Mean Divergence Measure for Elastic Image Registration[J]. Journal of Jilin University: Engineering and Technology Edition, 2009, 39(5): 1390-1392.

[8] CAO Hui. Research on Video-image Stabilization and Moving-Object Tracking in Ship Monitoring and Control[D]. Dalian: College of Marine Engineering, Dalian Maritime University, 2008.

[9] David G. Lowe. Object Recognition from Local Scale-invariant Features[C]. International Conference on Computer Vision, Corfu, Greece: Springer US, 1999: 1150-1157.

[10] David G. Lowe. Distinctive Image Features from Scale-invariant Keypoints[C]. International Conference on Computer Vision, Springer US, 2004: 91-110. 